\title{
Temozolomide-resistant Glioblastoma Depends on HDAC6 Activity Through Regulation of DNA Mismatch Repair
}

\author{
GO WOON KIM ${ }^{1 *}$, DONG HOON LEE ${ }^{1 *}$, SOO-KEUN YEON ${ }^{1}$, YU HYUN JEON ${ }^{1}$, \\ JUNG YOO ${ }^{1}$, SANG WOO LEE ${ }^{1}$ and SO HEE KWON ${ }^{1,2}$ \\ ${ }^{1}$ College of Pharmacy, Yonsei Institute of Pharmaceutical Sciences, Yonsei University, Incheon, Republic of Korea; \\ ${ }^{2}$ Department of Integrated OMICS for Biomedical Science, Yonsei University, Seoul, Republic of Korea
}

\begin{abstract}
Background/Aim: Histone deacetylase 6 (HDAC6) is considered as one of the most promising targets in drug development for cancer therapy. Drug resistance is a major cause of treatment failure in many cancers including glioblastoma (GBM), the most lethal malignant tumor. The role of HDAC6 in GBM resistance and its underlying mechanisms have not been well elucidated. Herein, we investigated the function of HDAC6 in modulating GBM resistance. Materials and Methods: The anticancer effects of four structurally distinct selective HDAC6 inhibitors were addressed using western blot, flow cytometry, CCK-8 assay, and CI in temozolomide (TMZ)-resistant GBM cells. Results: We showed that HDAC6-selecitve inhibitors block activation of the EGFR and p53 pathways in TMZ-resistant GBM cells. Importantly, the inhibition of HDAC6 correlates with increased levels of MSH2 and MSH6, key DNA mismatch repair proteins, in TMZ-resistant GBM cells. In addition to the MSH, HDAC6 inhibitors decrease MGMT expression in TMZresistant GBM cells. Furthermore, HDAC6 inhibitors increase TMZ sensitivity and efficiently induce apoptosis in TMZresistant GBM cells. Conclusion: Selective inhibition of HDAC6 may be a promising strategy for the treatment of TMZ-resistant GBM.
\end{abstract}

Glioblastoma (GBM), also known as World Health Organization grade IV glioma or glioblastoma multiforme, is the most common and lethal type of primary brain cancer

*These Authors contributed equally to this study.

Correspondence to: So Hee Kwon, College of Pharmacy, Yonsei Institute of Pharmaceutical Sciences, Yonsei University, 85 Songdogwahak-ro, Yeonsu-gu, Incheon, 21983, Republic of Korea. Tel: +82 327494513, Fax: +82 327494105, e-mail: soheekwon@yonsei.ac.kr

Key Words: Histone deacetylase 6, glioblastoma, temozolomide resistance, DNA mismatch repair, MutS $\alpha$.
$(1,2)$. With the introduction of combined chemotherapy and radiation therapy, the median survival time has increased to about 15 months (3). Temozolomide (TMZ) was used for the standard treatment of GBM for over a decade, irrespective of $\mathrm{O}^{6}$-methylguanine $\left(\mathrm{O}^{6}-\mathrm{MG}\right)$-DNA methyltransferase (MGMT) status $(4,5)$. However, almost all patients treated with TMZ eventually develop acquired resistance and tumor recurrence.

Several factors involved in epigenetic and genetic mechanisms have been associated with TMZ resistance. The mechanisms of resistance include increased DNA repair activity, overexpression of murine double minute 2 (MDM2), epidermal growth factor receptor (EGFR), and galetin-1; and mutations or alterations of phosphatase, tensin homolog (PTEN) and p53 (6). The MGMT DNA repair enzyme and DNA mismatch repair (MMR) system are the major known mechanisms of TMZ resistance in GBM (7-11). MGMT is a DNA repair protein, which removes the cytotoxic $\mathrm{O}^{6}-\mathrm{MG}$ DNA lesions made by TMZ, and high levels of MGMT expression are associated with TMZ resistance in GBM cells (8). The DNA MMR pathway is a system that corrects errors of nucleotide base mismatches made during DNA replication (12). Although there are six MutS homologs (MSH), MSH2 plays the major role in mismatch recognition, while other homologs such as MSH3 and MSH6 enhance the specificity of such recognition (13). A defective MMR system caused by mutations in MMR protein complexes leads to failure to recognize and repair $\mathrm{O}^{6}-\mathrm{MG}$ adducts generated by $\mathrm{TMZ}$, thereby making TMZ less effective $(6,14)$. Thus, strategies to rescue the effect of the MMR system need to improve the effect of TMZ and to overcome TMZ resistance.

Since the acetylation status of histone proteins and nonhistone proteins affects gene expression and protein activity in various biological processes, histone deacetylase (HDAC) inhibitors have emerged as a potential therapeutic approach to cancer $(15,16)$. However, because more than 11 classical HDACs (class I, II and IV HDACs) exist in human cells, the use of HDAC inhibitors targeting only a few types of HDACs 
for treatment of cancer or experimental studies is frequently more effective, with fewer side-effects (17). Class IIb HDAC6 has a unique structure (18). HDAC6 not only can function as a protein deacetylase, but also interacts with other regulatory proteins through ubiquitination and acetylationrelated mechanisms (19). HDAC6 is associated with cell sensitivity to drugs and emerging resistance during chemotherapy $(20,21)$. HDAC6 is overexpressed in GMB cell lines and tissues $(21,22)$, the overexpression of HDAC6 promotes GBM proliferation, and confers resistance to TMZ (21). Interestingly, HDAC6 and HDAC10 have a role in DNA MMR $(23,24)$. HDAC6 regulates MutS $\alpha$ by deacetylating and ubiquitinating MSH2 for degradation (24). HDAC6 significantly decreases sensitivity to DNA-damaging agents and decreases MMR activities by down-regulating MSH2. Additionally, MutS $\alpha$ inactivation is associated with TMZ resistance in $\operatorname{GBM}(9,11)$. However, little is known on the relevance of HDAC6, MGMT, and the MMR system to the development of TMZ resistance in GBM.

To shed more light on this issue, we investigated the functions of HDAC6 in GBM resistance using four structurally distinct HDAC6 selective inhibitors in TMZsensitive and TMZ-resistant GBM cells. Therefore, our findings provide a scientific rationale for targeting HDAC6 to overcome chemoresistance in TMZ-resistant GBM.

\section{Materials and Methods}

Cell culture. Human glioblastoma cell lines U87 (wtp53, no basal MGMT-TMZ sensitive cell), and T98G (mutp53, high basal MGMTTMZ resistant cell) were purchased from the American Type Culture Collection (Manassas, VA, USA). Cells were cultured in Dulbecco's modified Eagle's medium (Gibco; Thermo Fisher Scientific, Inc., Waltham, MA, USA) containing 10\% fetal bovine serum (FBS; HyClone; GE Healthcare, Logan, UT, USA), $100 \mathrm{U} / \mathrm{ml}$ penicillin, and $100 \mu \mathrm{g} / \mathrm{ml}$ streptomycin (Gibco; Thermo Fisher Scientific) in a humidified atmosphere of $5 \% \mathrm{CO}_{2}$ and $95 \%$ air at $37^{\circ} \mathrm{C}$.

Reagents. ACY-1215 (ricolinostat), CAY10603, temozolomide, and tubastatin A were purchased from Selleck Chemicals (Houston, TX, USA). A452 (purity 99\%) is a $\gamma$-lactam-based HDAC6 inhibitor (25) and was kindly provided by Dr. Gyoonhee Han (Yonsei University, Seoul, Republic of Korea).

Cell viability assay. Cell viability was assessed by measuring the dye absorbance of a water-soluble tetrazolium salt WST-8 [Cell Counting Kit (CCK)-8 kit, Dojindo Molecular Technologies, Kumamoto, Japan] according to the manufacturer's protocol and was performed as previously described (26). Cells were seeded in triplicates at a density of $1.5 \times 10^{3}$ cells in $200 \mu \mathrm{l}$ of medium in 96well plates. The drugs were added to the cells at the indicated concentrations $24 \mathrm{~h}$ after seeding at $37^{\circ} \mathrm{C}$ for $72 \mathrm{~h}$. The cells in each well were pulsed with $20 \mu \mathrm{l}$ of WST- 8 for the final $3 \mathrm{~h}$ of a $72-\mathrm{h}$ incubation, and absorbance was then measured at $450 \mathrm{~nm}$ using a multimode microplate reader (Tecan Group, Mannedorf, Switzerland). Absorbance was normalized to that of the negative control (no DMSO vehicle) at each time interval. To analyze cell viability, the percentage absorbance was calculated relative to negative control cultures. The results from three independent experiments performed in triplicate are presented.

Apoptosis assay. Apoptosis was assessed using Annexin $\mathrm{V} /$ propidium iodide (PI) double staining according to the manufacturer's protocol (FITC Annexin V Apoptosis Detection Kit; BD Biosciences, Franklin Lakes, NJ, USA), as previously described (26). The cells were then analyzed using a flow cytometer and BD FACSDiva software version 7 (both from BD Biosciences).

Western blot analysis. Cells grown and treated as indicated, were collected, lysed, and separated by sodium dodecyl sulfatepolyacrylamide gel electrophoresis (SDS-PAGE); western blotting was performed as previously described (27). The blots were semiquantified using FusionCapt software version 16.08a (Viber Lourmat Sté, Collégien, France). The protein expression levels were semi-quantified relative to GADPH and the levels in the $0.1 \%$ DMSO treated groups were set at 1 . GADPH was used as a loading control. The sources of the primary antibodies are available upon the request.

Drug combination analysis. For combined drug analysis, a constant ratio of TMZ and A452 or ACY-1215 was evaluated. Drug dilutions and combinations were prepared in media immediately prior to use. Cells $\left(1.5 \times 10^{3} /\right.$ well $)$ in 96 -well plates were incubated with the drugs for $72 \mathrm{~h}$ at $37^{\circ} \mathrm{C}$. A CCK-8 assay was performed to determine cell viability. Drug interactions were determined according to the combination index (CI) method described by Chou (28); CI $>1$ implies antagonism, $\mathrm{CI}=1$ is additive and $\mathrm{CI}<1$ implies synergism. CIs for the combination treatment groups were generated using CalcuSyn software version 2.11 (Biosoft, Cambridge, UK). The fraction affected (FA) was calculated from the percentage viability, as follows: $\mathrm{FA}=(100-$ percentage viability $) / 100$.

Statistical analysis. Statistical analyses were performed with GraphPad Prism software (version 7.0, Graphpad Software). Data are presented as the means \pm standard deviation of three independent experiments. Statistical differences were determined by one-way or two-way analysis of variance (ANOVA) with post-hoc analysis using Bonferroni's multiple comparison test. $p<0.05$ was considered to indicate a statistically significant difference.

\section{Results}

HDAC6-selective inhibitor activates MMR pathways in TMZ-resistant GBM cells but not in TMZ-sensitive cells. Loss of DNA MMR proteins is associated with GBM recurrence during TMZ treatment (29) and HDAC6 regulates MSH2 protein stability via de-acetylation and ubiquinination (24). Therefore, we assessed whether inhibition of HDAC6 could up-regulate MSH2 protein levels in GBM cells. To this end, we examined the expression level of MSH2 in TMZsensitive U87 and TMZ-resistant T98G GBM cells treated with four structurally distinct HDAC6-selective inhibitors, A452, ACY-1215, CAY10603, and tubastatin A. A452 and ACY-1215 resulted in slightly increased levels of MSH2 
A T98G (MGMT-proficient TMZ-resistant cells)
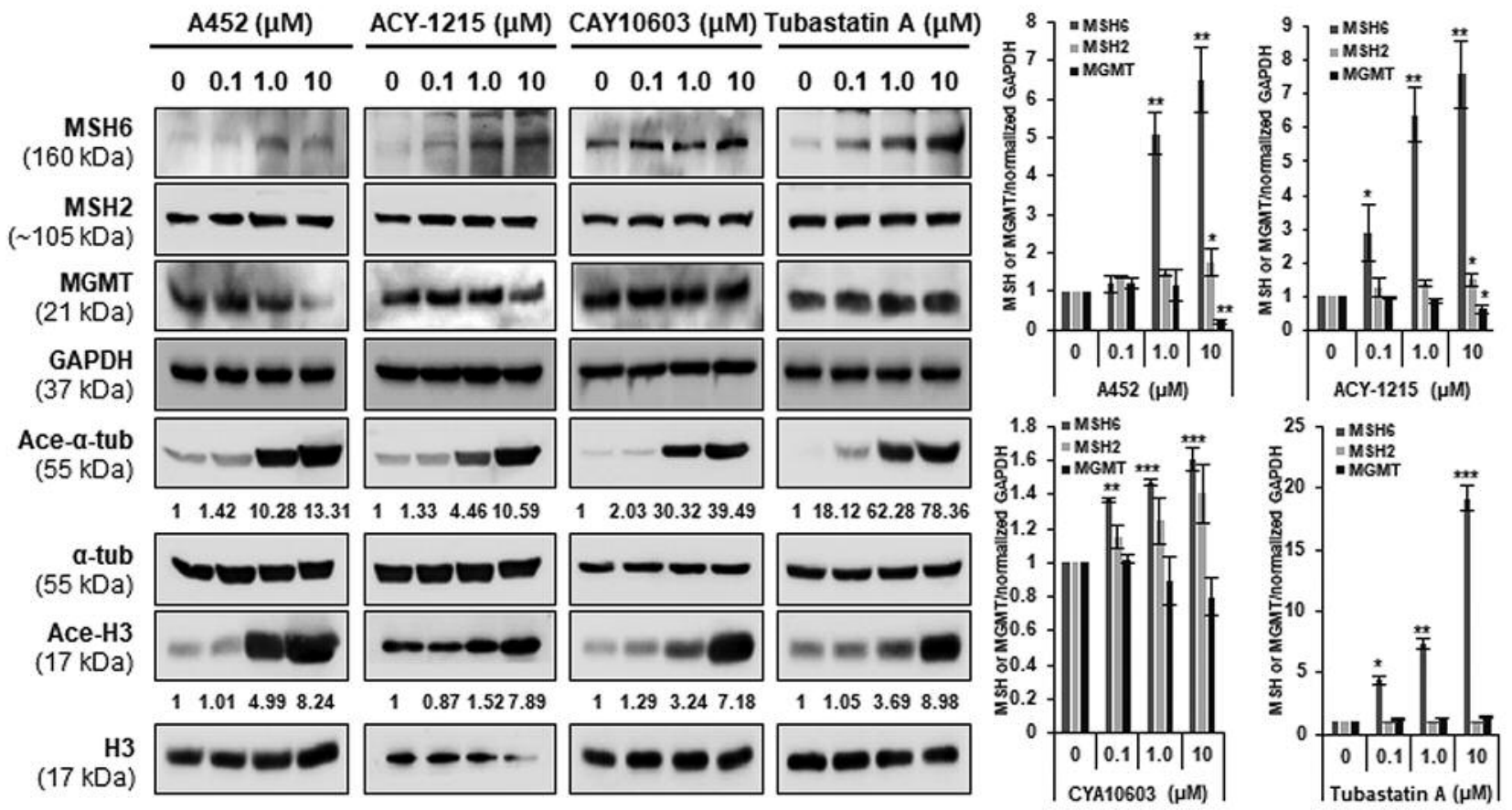

\section{B U87 (MGMT-deficient TMZ-sensitive cells)}
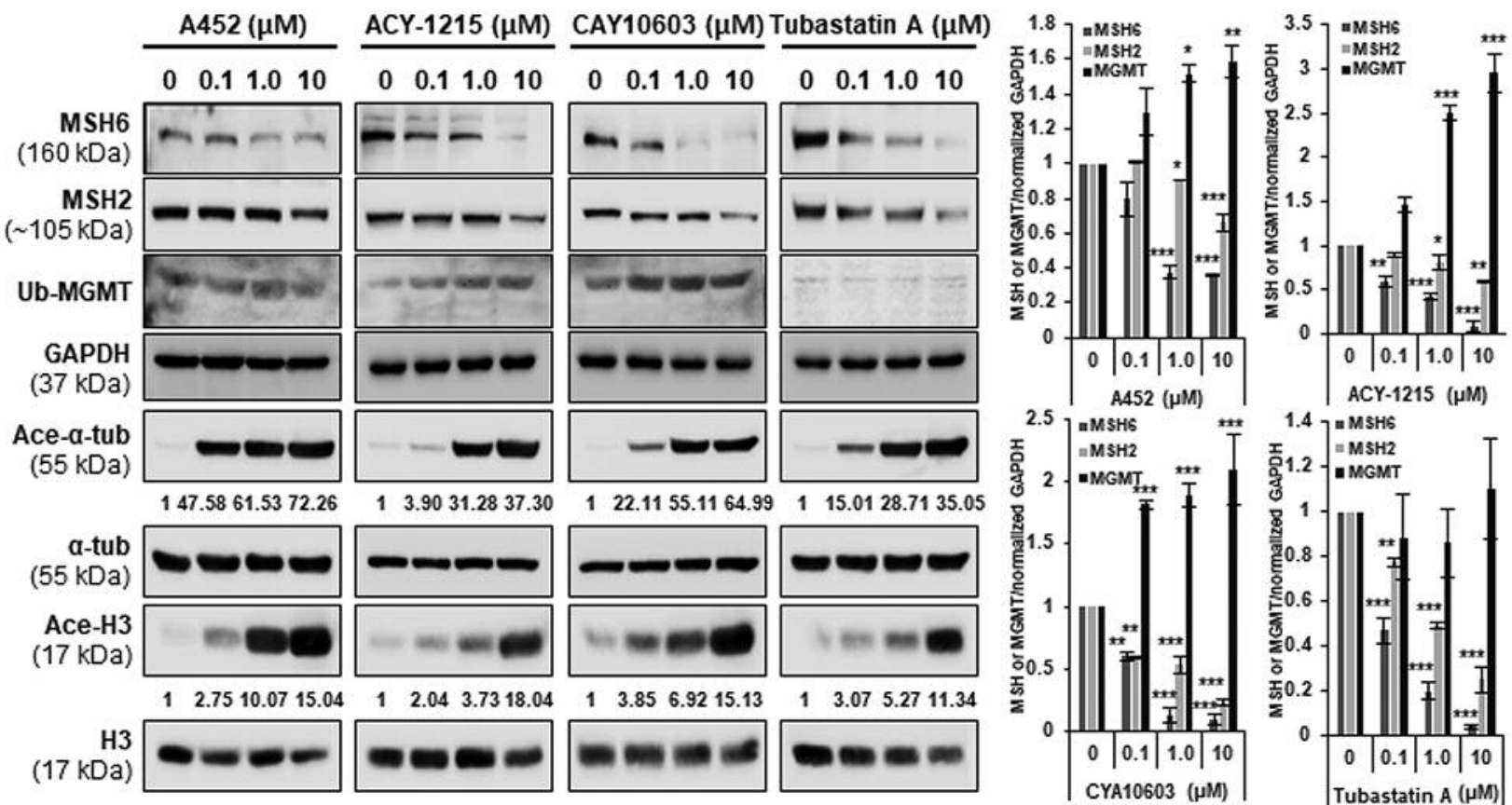

Figure 1. HDAC6 inhibitor up-regulates MSH2 and MSH6 proteins in TMZ-resistant GBM cells but not in TMZ-sensitive GBM cells. (A) TMZ-resistant T98G and (B) TMZ-sensitive U87 GBM cells were treated with $0.1 \%$ DMSO (control) or HDAC6 inhibitor at the indicated concentrations (0.1, 1, and $10 \mu \mathrm{M})$ for $24 \mathrm{~h}$. Whole-cell lysates were subjected to immunoblotting with the indicated antibodies. Acetylation of H3 (Ace-H3) and $\alpha$-tubulin (Ace$\alpha$-tub) are markers for HDAC1 and HDAC6 inhibition, respectively. The protein expression levels were semi-quantified relative to GAPDH, and the levels in the 0.1\% DMSO-treated groups were set at 1. Levels of Ace- $\alpha$-tub and Ace-H3 were semi-quantified relative to $\alpha$-tub and H3, respectively; GAPDH, $\alpha$-tub and histone H3 were used as equal loading controls. ${ }^{*} p<0.05, * * p<0.01$, and ${ }^{* * *} p<0.001$ vs. the DMSO control group. 


\section{A T98G (MGMT-proficient TMZ-resistant cells)}

\begin{tabular}{|c|c|c|c|c|}
\hline & A452 $(\mu \mathrm{M})$ & ACY-1215 $(\mu \mathrm{M})$ & CAY10603 $(\mu \mathrm{M})$ & Tubastatin $\mathrm{A}(\mu)$ \\
\hline & $\begin{array}{llll}0 & 0.1 & 1.0 & 10\end{array}$ & $\begin{array}{llll}0 & 0.1 & 1.0 & 10\end{array}$ & $\begin{array}{llll}0 & 0.1 & 1.0 & 10\end{array}$ & $\begin{array}{lllll}0 & 0.1 & 1.0 & 10\end{array}$ \\
\hline $\begin{array}{r}\text { pEGFR } \\
(170 \mathrm{kDa})\end{array}$ & $\mathbf{m}-\mathbf{m}$ & $=-m$ & 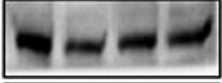 & 110 \\
\hline $\begin{array}{r}\text { EGFR } \\
(170 \mathrm{kDa})\end{array}$ & $-\infty-\infty$ & $--\infty-$ & $n=-\pi$ & 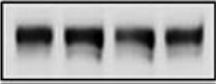 \\
\hline $\begin{array}{r}\text { GAPDH } \\
(37 \mathrm{kDa})\end{array}$ & 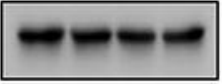 & $-\infty$ & & 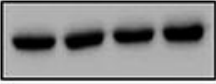 \\
\hline $\begin{array}{r}\text { Ace-a-tub } \\
(55 \mathrm{kDa})\end{array}$ & & & - & \\
\hline $\begin{array}{r}\text { a-tub } \\
(55 \mathrm{kDa})\end{array}$ & $--\infty$ & $-\infty$ & 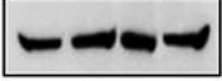 & - \\
\hline
\end{tabular}

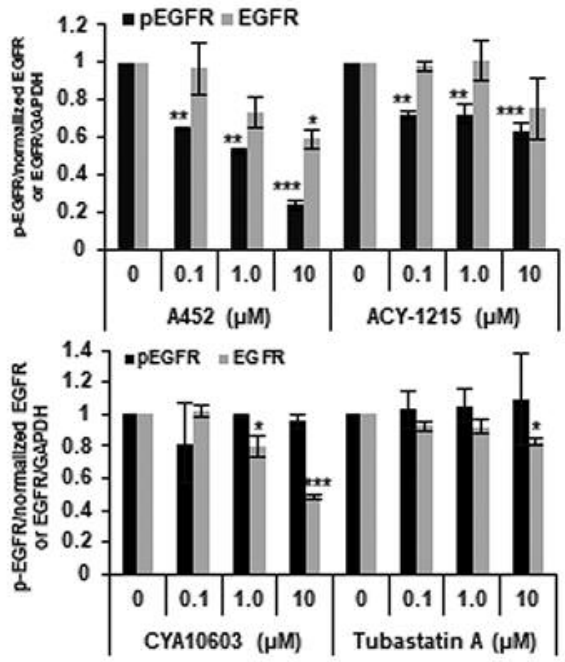

B U87 (MGMT-deficient TMZ-sensitive cells)
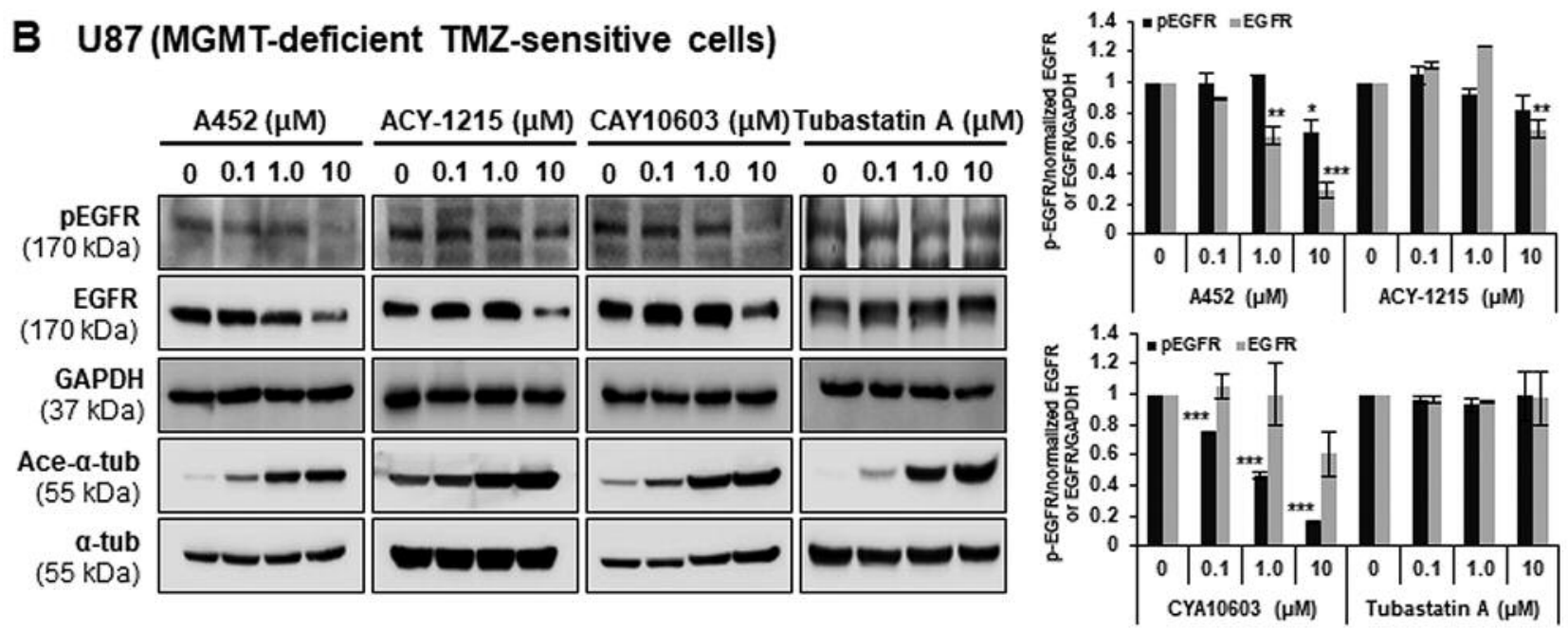

Figure 2. HDAC6 inhibitors lead to a pronounced inhibition of EGFR in both TMZ-resistant and TMZ-sensitive GBM cells. (A) TMZ-resistant T98G and (B) TMZ-sensitive U87 GBM cells were treated with $0.1 \%$ DMSO (control) or HDAC6 inhibitor at the indicated concentrations for 24 h. Wholecell lysates were subjected to immunoblotting with the indicated antibodies. Relative protein expression levels were semi-quantified by densitometric analysis of the blots. pEGFR was semiquantified relative to EGFR. Levels of Ace- $\alpha$-tub were semiquantified relative to $\alpha$-tub; GAPDH and $\alpha$-tub were used as equal loading controls. ${ }^{*} p<0.05$, ** ${ }^{*}<0.01$, and $* * * p<0.001$ vs. the DMSO control group.

protein in TMZ-resistant T98G cells (Figure 1A). Previous studies suggest that MSH6 stabilizes MSH2 by forming MSH2-MSH6 heterodimers (30). Thus, we examined whether HDAC6 inhibitors could up-regulate MSH6 protein levels. In addition to MSH2, four HDAC6 inhibitors substantially increased MSH6 levels in TMZ-resistant T98G cells. In contrast, all tested HDAC6 inhibitors decreased the levels of both MSH6 and, to a lesser extent, MSH2 proteins in TMZ-sensitive U87 cells (Figure 1B). These results indicate that selective inhibition of HDAC6 markedly increases MSH protein levels in TMZ-resistant GBM cells, but not in TMZ-sensitive GBM cells.
Next, we investigated whether HDAC6 inhibitor affects the expression of another DNA repair protein, MGMT, related to DNA MMR and TMZ resistance. As shown in Figure 1A, A452 and ACY-1215 reduced MGMT levels in TMZ-resistant T98G cells with high basal MGMT expression. On the other hand, TMZ-sensitive U87 cells with no MGMT expression due to gene silencing by promoter methylation appeared ubiquitinated MGMT, but not unmodified MGMT. A452, ACY-1215 and CAY10603 slightly increased the levels of ubiquitinated MGMT. No detectable alterations in MGMT protein level were observed in TMZ-sensitive U87 cells treated with tubastatin A (Figure 

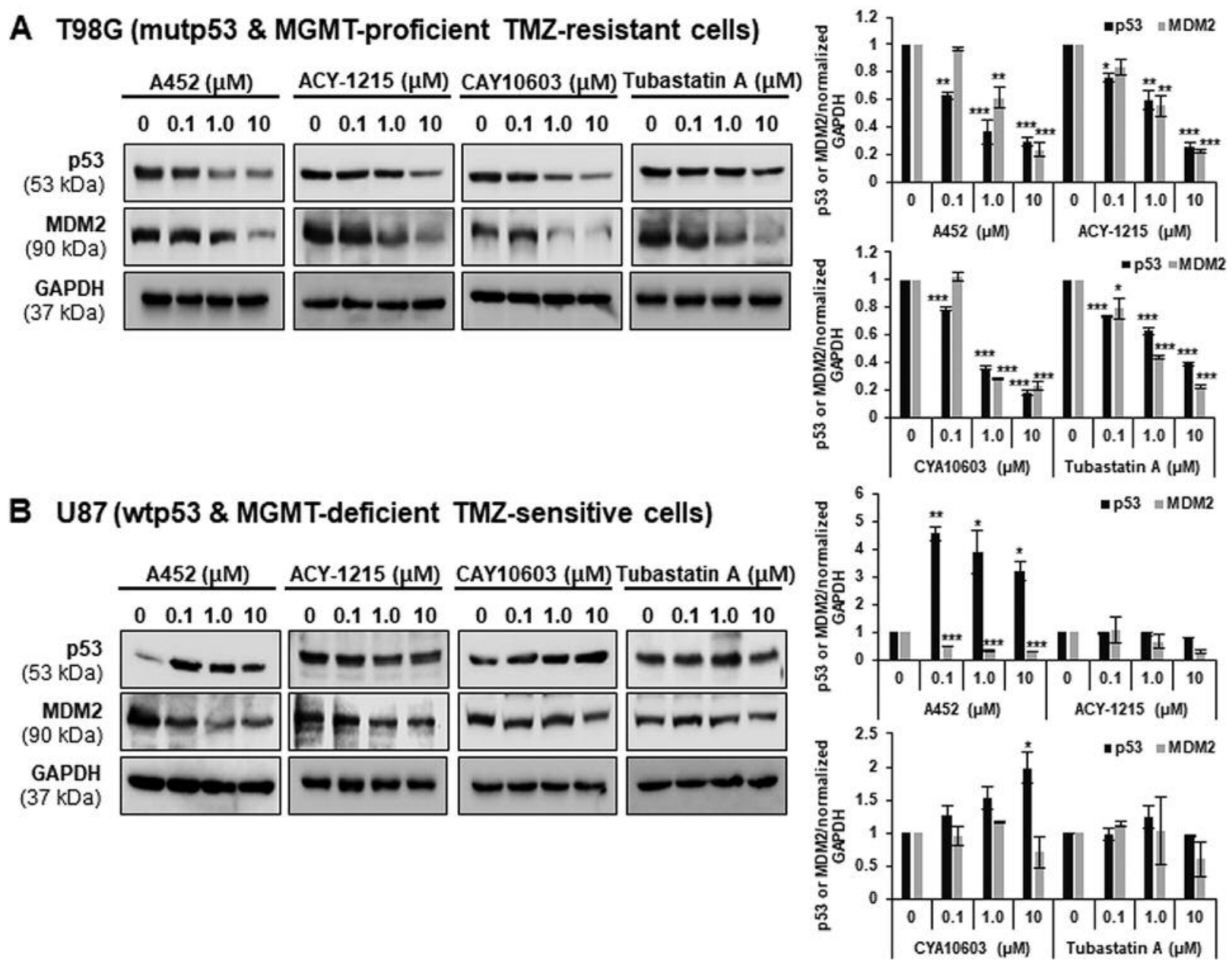

Figure 3. HDAC6 inhibitor differentially modulates p53 in both TMZ-resistant and TMZ-sensitive GBM cells. (A) TMZ-resistant T98G and (B) TMZsensitive U87 GBM cells were treated with $0.1 \%$ DMSO (control) or HDAC6 inhibitor at the indicated concentrations for 24 h. Whole-cell lysates were subjected to immunoblotting with the indicated antibodies. ${ }^{*} p<0.05, * * p<0.01$, and ${ }^{* * *} p<0.001$ vs. the DMSO control group.

1B). Overall, this result indicates that HDAC6-selective inhibitors modulate MGMT protein levels in TMZ-resistant GBM cells, but not in TMZ-sensitive GBM cells.

HDAC6-selective inhibitor inactivates EGFR pathway by destabilizing EGFR in GBM cells. Previous studies have shown that EGFR plays a pivotal role in TMZ resistance (31) and HDAC6 inhibitors inactivate the EGFR pathway (21). To test whether HDAC6 inhibitors regulate EGFR in TMZsensitive and TMZ-resistant GBM cells, we tested the phosphorylation level of EGFR and total EGFR by western blotting. A452, ACY-1215 and CAY10603 destabilized EGFR at higher concentrations in TMZ-sensitive U87 and TMZ-resistant T98G GBM cells (Figure 2). Moreover, inhibition of HDAC6 by these three HDAC6-selective inhibitors decreased EGFR phosphorylation levels in both
GBM cells. This finding suggests that inhibition of HDAC6 controls the survival and chemoresistance of GBM cells via destabilization of EGFR and inactivation of the EGFR pathway.

HDAC6-selective inhibitor differentially modulates p53 by up-regulating wild-type and down-regulating mutant p53 in GBM cells. Previously, we reported that inhibition of HDAC6 by A452 differentially modulates p53 by upregulating wtp53 and down-regulating mutp53 in cancer cells (32). In the present study, we assessed whether HDAC6 altered the p53 signaling pathway in TMZ-sensitive and TMZ-resistant GBM cells. Four tested HDAC6 inhibitors robustly down-regulated mutp53 protein in TMZ-resistant T98G cells in a dose-dependent manner (Figure 3A). Conversely, A452 and CAY10603 slightly up-regulated 
wtp53 protein in TMZ-sensitive U87 cells (Figure 3B). MDM2, a key negative regulator of p53, down-regulated in GBM cells, independently of p53 and TMZ resistance status.

HDAC6-selective inhibitor sensitizes GBM cells to TMZ. Among four tested HDAC6 inhibitors, we selected and used for further study the two more effective HDAC6 inhibitors, A452 and ACY-1215, based on MutS $\alpha$ and EGFR destabilization result. Next, we examined the effects of the two HDAC6 inhibitors, A452 and ACY-1215, on cell viability in TMZ-sensitive and TMZ-resistant GBM cells. Cells were cultured with an HDAC6 inhibitor for up to $72 \mathrm{~h}$, and cell viability was measured by CCK-8 assays. A452 and ACY-1215 resulted in a dose-dependent decrease in cell viability in both TMZ-sensitive U87 and TMZ-resistant T98G GBM cells (Figure 4). Next, we tested whether HDAC6 inhibitor enhanced the antiproliferative effects of TMZ in TMZ-sensitive and TMZ-resistant GBM cells. The combination of A452 with TMZ augmented cell death compared with cultures treated with TMZ alone in both cells (Figure 4). Similarly, the combination of TMZ and ACY-1215 showed synergistic cytotoxicity. Furthermore, the CI values, a quantitative measure of drug interaction according to the Chou and Talalay method, were evaluated (28). The CI for A452-TMZ and ACY-1215-TMZ in both GBM cells was $<1$, indicating the synergistic effect of the combination (Figure 4). In particular, A452 demonstrated significant synergy in the TMZ-resistant T98G GBM cells compared with TMZ-sensitive U87 GBM cells. The ACY1215-TMZ combination exhibited similar synergistic effects on both GBM cells, albeit weaker synergy than the A452-TMZ combination. This finding indicates that the HDAC6 inhibitorTMZ combination may be more efficient on TMZ-resistant GBM cells with mutp53 and MGMT expression and that the HDAC6 inhibitor may overcome TMZ resistance.

HDAC6-selective inhibitor in combination with TMZ synergistically increase apoptosis in TMZ-resistant GMB cells. Selective inhibition of HDAC6 increased MMR proteins in TMZ-resistant T98G cells, but not in TMZsensitive U87 GBM cells. Therefore, we focused on T98G cells which express MGMT and have mutated p53. To investigate the mechanism of cell death in GBM cells cultured with a combination of HDAC6 inhibitor and TMZ, we evaluated their apoptotic effects. Cells treated with the combination of TMZ and A452 or ACY-1215 had increased levels of cleaved poly(ADP ribose) polymerase (PARP) and of active caspase-3, which are apoptosis markers (Figure 5A). Furthermore, Annexin V/PI staining revealed that cell apoptosis was significantly increased following combination treatment of cells with TMZ and A452 or ACY-1215 (Figure 5B). Overall, our results suggest that combination treatment with an HDAC6-selective inhibitor and TMZ triggers apoptosis by activating caspase 3 .

\section{Discussion}

The ultimate hindrance to the clinical success of GBM treatment is drug resistance causing high recurrence rate of this cancer. HDACs, the most promising target in drug development for cancer therapy, are known to be associated with oncogenesis of GBM in various human GBM cell lines (33). In this paper, we analyzed the effects of treatment of HDAC6-selective inhibitors in order to find potential relationship between HDAC6 and GBM resistance. Thus, we conducted further research into the detailed mechanism of HDAC6 inhibition and the potential of combination of treatment of HDAC6-selective inhibitor with TMZ, a widely used chemotherapeutic drug.

We have shown the different impact of each HDAC6 inhibitor on GBM proliferation and resistance by differential modulation of DNA MMR, EGFR, and p53 pathways. EGFR targeting has been studied as a cancer treatment strategy. Reducing EGFR protein levels as well as inhibiting EGFR kinase activity is a pivotal strategy to block the oncogenic effects of EGFR in cells. HDAC6 deacetylates the molecular chaperone Hsp90, and the deacetylated active form of Hsp90, regulates its chaperone activity for its client proteins, including EGFR $(34,35)$. Thus, the use of HDAC6 inhibitors is another strategy to down-regulate EGFR protein levels in cancer cells. Overexpression of EGFR is also associated with chemoresistance in GBM (31). In consistent with previous results, EGFR expression was higher in TMZ-resistant GBM cells than TMZ-sensitive GBM cells. First, we showed that HDAC6 inhibitors reduced the phosphorylation levels of EGFR and total EGFR in both TMZ-sensitive and TMZ-resistant GBM cells although the altered levels of EGFR were different. This result suggests that selective inhibition of HDAC6 controls the survival and chemoresistance of GBM cells by destabilizing EGFR and inactivating the EGFR pathway.

Figure 4. Combined treatment of HDAC6 inhibitor and TMZ triggers synergistic cytotoxicity. (A) TMZ-resistant T98G and (B) TMZ-sensitive U87 GBM cells were treated with $0.1 \%$ DMSO (control) or the indicated compounds alone or in combination for $72 \mathrm{~h}$. Combination treatments were then performed in cells maintaining a constant ratio between the doses of TMZ and A452 or ACY-1215 and cell viability was assessed at $72 \mathrm{~h}$ by CCK-8 assay. The combination index (CI) value and the relative fraction affected (FA) were determined at each dose combination (actual) and a simulation was run to estimate the CI values and confidence intervals across the entire FA range (simulation). $C I<1$, $C I=1$ and $C I>1$ indicate synergistic, additive and antagonistic effects, respectively. Significance was determined by two-way analysis of ANOVA with Bonferroni's post-hoc test to compare groups. ${ }^{*} p<0.05$,

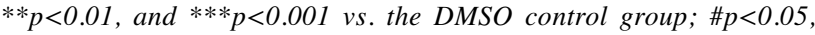

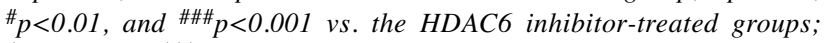
$\$ p<0.05$ and $\$ \$ \$ p<0.001$ vs. the TMZ-treated group. 


\section{A T98G (MGMT-proficient TMZ-resistant cells)}
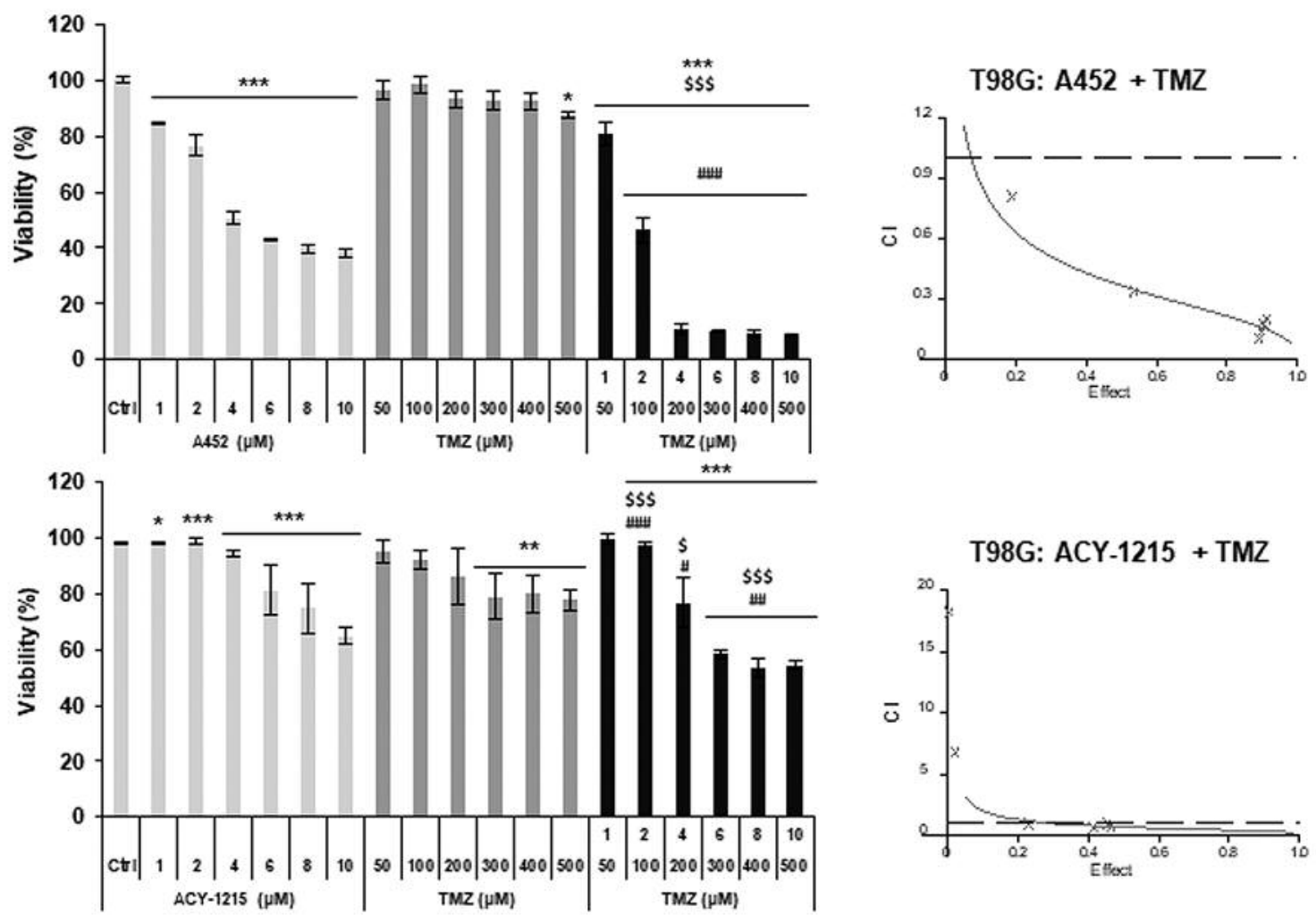

B U87 (MGMT-deficient TMZ-sensitive cells)
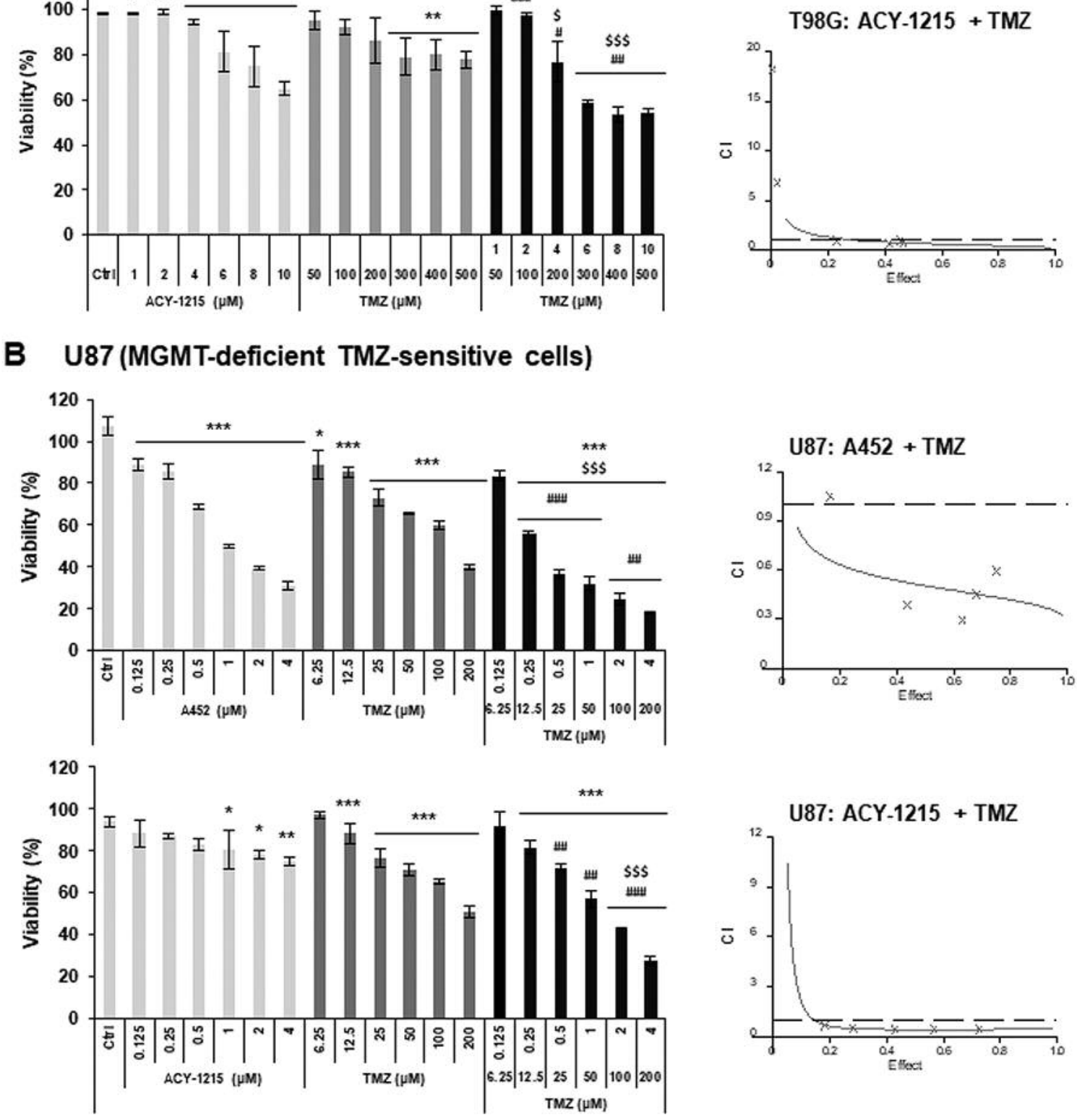


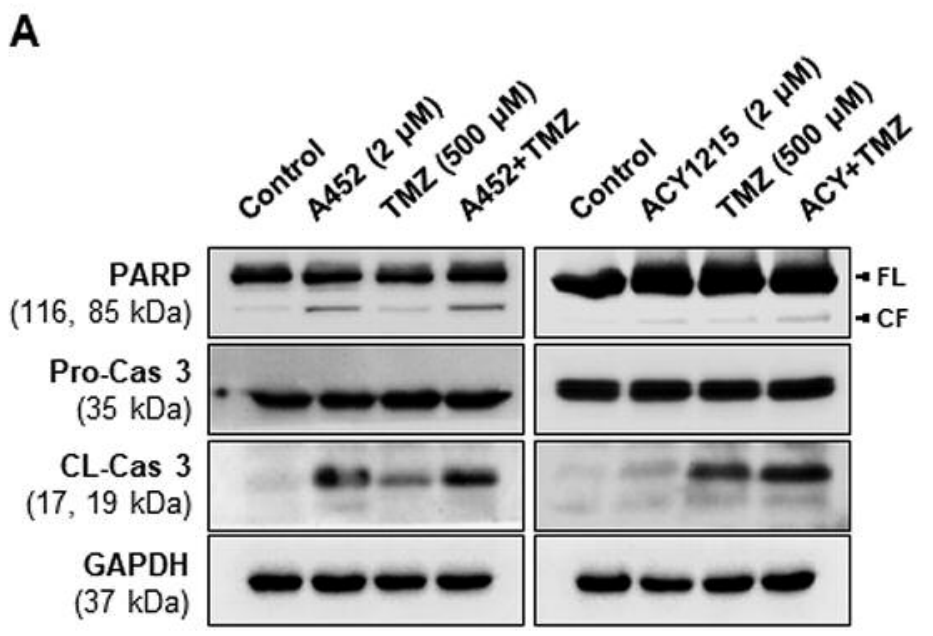

B

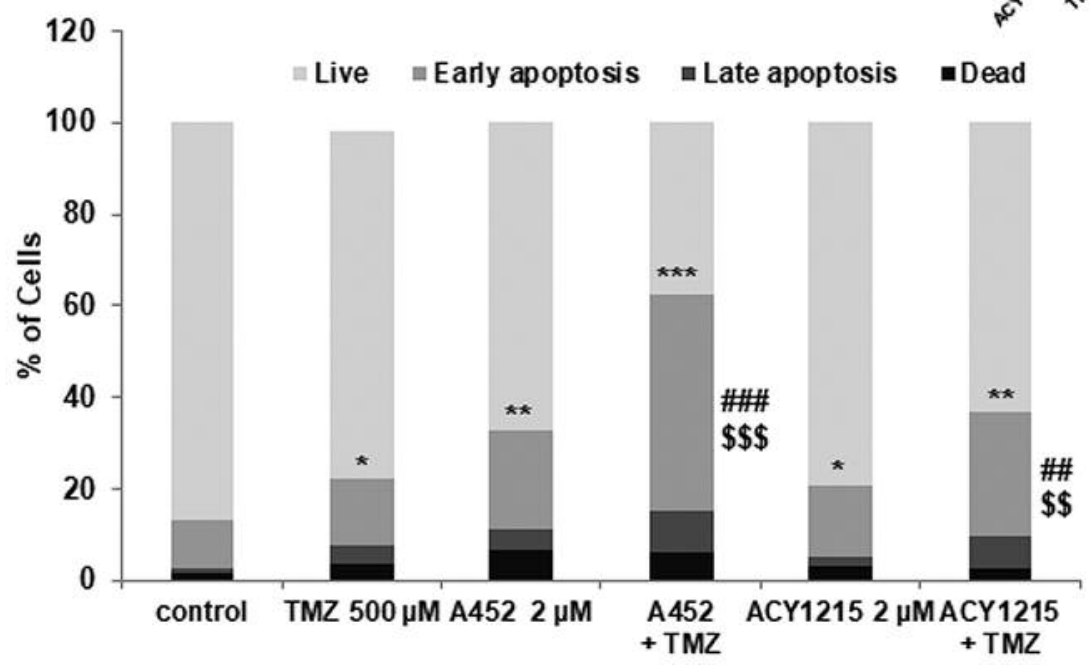

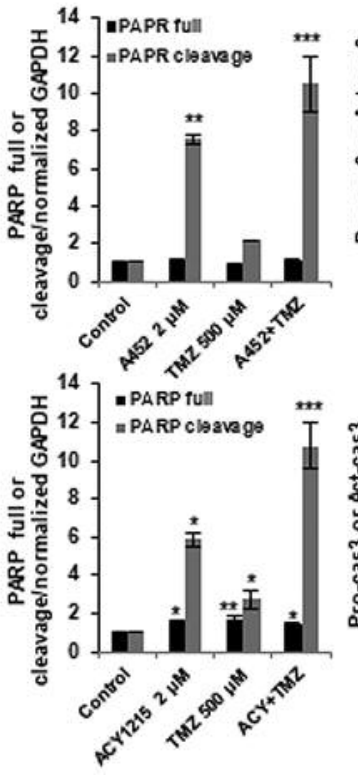

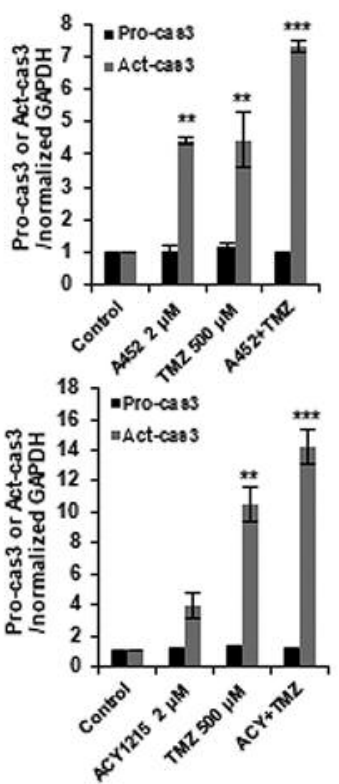

Figure 5. HDAC6 inhibitors in combination with TMZ synergistically increase apoptosis in TMZ-resistant T98G cells. (A) T98G cells were treated with $0.1 \%$ DMSO (control), TMZ, A452, or ACY-1215, or in combination with these compounds for 24 h. Whole-cell lysates were subjected to immunoblotting with the indicated antibodies. $* p<0.05$, $* * p<0.01$, and $* * * p<0.001 \mathrm{vs}$. the DMSO control group. (B) TMZ-resistant T98G cells were treated with $0.1 \%$ DMSO (control), TMZ, A452, or ACY-1215 or in combination with these compounds for 48 h. Cell death was assessed by flow cytometry and Annexin V/PI staining ( $n=3$ ). Significance was determined by two-way analysis of ANOVA with Bonferroni's post-hoc test to

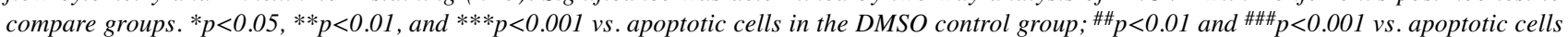
in the HDAC6 inhibitor-treated group; ${ }^{\$} p<0.01$ and $\$ \$ \$ p<0.001$ vs. the TMZ-treated group.

Second, we demonstrated that four tested HDAC6 inhibitors robustly down-regulated mutp53 protein in TMZresistant T98G cells. In contrast, A452 and CAY10603 upregulated wtp53 protein in TMZ-sensitive U87 cells. Although mutp53 is associated with resistance to TMZ and most studied, the results have been mixed. Furthermore, p53 status was not always correlated with MGMT status in GBM cell lines; TMZ-sensitive GBM cells (e.g., U87, A172) possess wtp53 and p53 gene mutations were found in TMZ- resistant GBM cells (e.g., $\mathrm{LN}-18, \mathrm{~T} 98 \mathrm{G}, \mathrm{U} 138)$ as well as TMZ sensitive-GBM cells (e.g., U251, U373). Thus, these data indicate that a mutation in the TP53 gene does not seem to be a primary indicator of resistance to TMZ (36). MDM2, which is a p53-negative regulator related to resistant GBM cell lines, decreased tumor size when down-regulated with the antisense oligonucleotide (37). Interestingly, HDAC6 inhibitors caused decreased levels of MDM2 in both TMZsensitive and TMZ-resistant GBM cells, independently of 
their p53 and TMZ resistance status. This finding indicates that HDAC6-selective inhibitors may cause cell death by reduction of hyperstable mutp53 as an oncogene.

Third, we found that HDAC6-selective inhibitor activates MMR pathways in TMZ-resistant cells, but not in TMZsensitive cells. In line with previous findings, HDAC6selective inhibitors increased the levels of MSH2 protein in TMZ-resistant GBM cells. Zhang $\mathrm{M}$ et al. have reported that HDAC6 interacts with MSH2 and MSH6, deacetylating these MMR proteins. Subsequently, the deacetylated MSH2 is degraded by proteasome, reducing DNA MMR activity (24). However, they did not provide the evidence that MSH6 protein stability can be directly regulated by HDAC6. In our finding, HDAC6-selective inhibitors significantly increased the protein levels of MSH6 as well as MSH2 in TMZ-resistant GBM cells. In contrast, the effect of HDAC6 inhibitor on activation of the MMR pathway was not observed in TMZ-sensitive GBM cells, probably because MSH2 and MSH6 are already highly expressed in TMZ-sensitive cells. It has also been reported that mutation of MSH6 occurs after TMZ treatment and knockdown of MSH6 increases TMZ resistance in U251 GBM cells (38). In addition, the MSH6 mutation was mostly found in recurrent GBM patients, but not in newlydiagnosed GBM patients (39). Based on these findings, MSH6 may play a crucial role in TMZ resistance in GBM. Since our findings show that HDAC6 regulates MSH6 levels, HDAC6 inhibitor may be a potential therapeutic agent to overcome TMZ resistance.

Thus, further work will be required to determine the molecular mechanisms whereby HDAC6 regulates MSH6 stability.

Another DNA repair protein, MGMT, which is related to DNA MMR and TMZ resistance, was reduced by treatment with A452 and ACY-1215 in TMZ-resistant GBM cells. These results are hopeful for overcoming TMZ-resistance and, perhaps, suggest a possible utility of the inhibition of HDAC6 in combination with traditional chemotherapy for GBM. Indeed, HDAC6 sensitized GBM cells to the inhibition of TMZ-induced cell proliferation and the induction of apoptosis, which occur as a result of the activation of caspases and DNA MMR and the destabilization of EGFR and p53 in TMZ-resistant GBM cells. Our findings indicate that HDAC6-mediated destabilization of MutS $\alpha$ and stabilization of MGMT may partly explain the oncogenic function of HDAC6 in GBM resistance. Taken together, our results suggest that selective inhibition of HDAC6 may be a promising strategy for the treatment of GBM and overcoming $\mathrm{TMZ}$ resistance.

\section{Conflicts of Interest}

The Authors report no conflicts of interest regarding this study.

\section{Authors' Contributions}

GWK and DHL designed and performed experiments, as well as analyzed the data. SKY, YHJ, YJ, and SWL performed experiments. S.H.K conceived the general design of the study, participated in the development of the approaches, wrote the initial draft of the manuscript, extensively edited the manuscript and supervised the work.

\section{Acknowledgements}

The Authors would like to thank Dr. Gyoonhee Han (Yonsei University, Seoul, Korea) for providing A452. This research was supported by the Basic Science Research Program through the National Research Foundation of Korea (NRF) funded by the Ministry of Education, Science and Technology (2016R1D1A1A 02937071, 2018R1A6A1A03023718, 2019R1I1A1A01058601, and 2019R1A2C1008619).

\section{References}

$1 \mathrm{Ng} \mathrm{K}$, Kim R, Kesari S, Carter B and Chen CC: Genomic profiling of glioblastoma: Convergence of fundamental biologic tenets and novel insights. J Neurooncol 107(1): 1-12, 2012. PMID: 22002595. DOI: 10.1007/s11060-011-0714-2

2 Rich JN and Bigner DD: Development of novel targeted therapies in the treatment of malignant glioma. Nat Rev Drug Discov 3(5): 430-446, 2004. PMID: 15136790. DOI: $10.1038 / \operatorname{nrd} 1380$

3 Stupp R, Pavlidis N, Jelic S and Force EGT: Esmo minimum clinical recommendations for diagnosis, treatment and followup of malignant glioma. Ann Oncol 16(Suppl 1): i64-65, 2005. PMID: 15888760 . DOI: 10.1093/annonc/mdi834

4 Stupp R, Brada M, van den Bent MJ, Tonn JC, Pentheroudakis $\mathrm{G}$ and Group EGW: High-grade glioma: Esmo clinical practice guidelines for diagnosis, treatment and follow-up. Ann Oncol 25(Suppl 3): iii93-101, 2014. PMID: 24782454. DOI: 10.1093/ annonc/mdu050

5 Mellai M1, Caldera V, Annovazzi L, Chiò A, Lanotte M, Cassoni $P$, Finocchiaro $G$ and Schiffer D: MGMT promoter hypermethylation in a series of 104 glioblastomas. Cancer Genomics Proteomics 6(4): 219-227, 2009. PMID: 19656999.

6 Messaoudi K, Clavreul A and Lagarce F: Toward an effective strategy in glioblastoma treatment. Part i: Resistance mechanisms and strategies to overcome resistance of glioblastoma to temozolomide. Drug Discov Today 20(7): 899905, 2015. PMID: 25744176. DOI: 10.1016/j.drudis.2015.02.011

7 Cahill DP, Codd PJ, Batchelor TT, Curry WT and Louis DN: Msh6 inactivation and emergent temozolomide resistance in human glioblastomas. Clin Neurosurg 55: 165-171, 2008. PMID: 19248684.

8 Kitange GJ, Carlson BL, Schroeder MA, Grogan PT, Lamont JD, Decker PA, Wu W, James CD and Sarkaria JN: Induction of mgmt expression is associated with temozolomide resistance in glioblastoma xenografts. Neuro Oncol 11(3): 281-291, 2009. PMID: 18952979. DOI: 10.1215/15228517-2008-090

9 McFaline-Figueroa JL, Braun CJ, Stanciu M, Nagel ZD, Mazzucato P, Sangaraju D, Cerniauskas E, Barford K, Vargas A, Chen Y, Tretyakova N, Lees JA, Hemann MT, White FM and 
Samson LD: Minor changes in expression of the mismatch repair protein msh2 exert a major impact on glioblastoma response to temozolomide. Cancer Res 75(15): 3127-3138, 2015. PMID: 26025730. DOI: 10.1158/0008-5472.CAN-14-3616

10 Shinsato Y, Furukawa T, Yunoue S, Yonezawa H, Minami K, Nishizawa Y, Ikeda R, Kawahara K, Yamamoto M, Hirano H, Tokimura $\mathrm{H}$ and Arita K: Reduction of mlh1 and pms2 confers temozolomide resistance and is associated with recurrence of glioblastoma. Oncotarget 4(12): 2261-2270, 2013. PMID: 24259277. DOI: 10.18632/oncotarget.1302

11 Stark AM, Doukas A, Hugo HH, Hedderich J, Hattermann K, Maximilian Mehdorn $\mathrm{H}$ and Held-Feindt J: Expression of DNA mismatch repair proteins mlh1, msh2, and msh6 in recurrent glioblastoma. Neurol Res 37(2): 95-105, 2015. PMID: 24995467. DOI: 10.1179/1743132814Y.0000000409

12 Fishel R and Wilson T: Muts homologs in mammalian cells. Curr Opin Genet Dev 7(1): 105-113, 1997. PMID: 9024626. DOI: $10.1016 / \mathrm{s} 0959-437 \times(97) 80117-7$

13 Jiricny $\mathrm{J}$ and Nystrom-Lahti M: Mismatch repair defects in cancer. Curr Opin Genet Dev 10(2): 157-161, 2000. PMID: 10753784. DOI: $10.1016 / \mathrm{s} 0959-437 x(00) 00066-6$

14 Liu L, Markowitz S and Gerson SL: Mismatch repair mutations override alkyltransferase in conferring resistance to temozolomide but not to 1,3-bis(2-chloroethyl)nitrosourea. Cancer Res 56(23): 5375-5379, 1996. PMID: 8968088.

15 Marks P, Rifkind RA, Richon VM, Breslow R, Miller T and Kelly WK: Histone deacetylases and cancer: Causes and therapies. Nat Rev Cancer 1(3): 194-202, 2001. PMID: 11902574. DOI: $10.1038 / 35106079$

16 Garmpis N, Damaskos C, Garmpi A, Kalampokas E, Kalampokas T, Spartalis E, Daskalopoulou A, Valsami S, Kontos M, Nonni A, Kontzoglou K, Perrea D, Nikiteas N, Dimitroulis D: Histone deacetylases as new therapeutic targets in triple-negative breast cancer: Progress and promises. Cancer Genomics Proteomics 14(5): 299-313, 2017. PMID: 28870998. DOI: $10.21873 / \mathrm{cgp} .20041$

17 Gupta PK, Reid RC, Liu L, Lucke AJ, Broomfield SA, Andrews MR, Sweet MJ and Fairlie DP: Inhibitors selective for hdac6 in enzymes and cells. Bioorg Med Chem Lett 20(23): 7067-7070, 2010. PMID: 20947351. DOI: 10.1016/j.bmcl.2010.09.100

18 Seigneurin-Berny D, Verdel A, Curtet S, Lemercier C, Garin J, Rousseaux S and Khochbin S: Identification of components of the murine histone deacetylase 6 complex: Link between acetylation and ubiquitination signaling pathways. Mol Cell Biol 21(23): 80358044, 2001. PMID: 11689694. DOI: 10.1128/MCB.21.23.80358044.2001

19 Lee YS, Lim KH, Guo X, Kawaguchi Y, Gao Y, Barrientos T, Ordentlich P, Wang XF, Counter CM and Yao TP: The cytoplasmic deacetylase hdac6 is required for efficient oncogenic tumorigenesis. Cancer Res 68(18): 7561-7569, 2008. PMID: 18794144. DOI: 10.1158/0008-5472.CAN-08-0188

20 Ai J, Wang Y, Dar JA, Liu J, Liu L, Nelson JB and Wang Z: Hdac6 regulates androgen receptor hypersensitivity and nuclear localization via modulating hsp90 acetylation in castrationresistant prostate cancer. Mol Endocrinol 23(12): 1963-1972, 2009. PMID: 19855091. DOI: 10.1210/me.2009-0188

21 Wang Z, Hu P, Tang F, Lian H, Chen X, Zhang Y, He X, Liu W and Xie C: Hdac6 promotes cell proliferation and confers resistance to temozolomide in glioblastoma. Cancer Lett 379(1): 134-142, 2016. PMID: 27267806. DOI: 10.1016/j.canlet. 2016.06 .001
22 Aldape K, Zadeh G, Mansouri S, Reifenberger G and von Deimling A: Glioblastoma: Pathology, molecular mechanisms and markers. Acta Neuropathol 129(6): 829-848, 2015. PMID: 25943888. DOI: 10.1007/s00401-015-1432-1

23 Radhakrishnan R, Li Y, Xiang S, Yuan F, Yuan Z, Telles E, Fang J, Coppola D, Shibata D, Lane WS, Zhang Y, Zhang X and Seto E: Histone deacetylase 10 regulates DNA mismatch repair and may involve the deacetylation of muts homolog 2. J Biol Chem 290(37): 22795-22804, 2015. PMID: 26221039. DOI: 10.1074/jbc.M114.612945

24 Zhang M, Xiang S, Joo HY, Wang L, Williams KA, Liu W, Hu C, Tong D, Haakenson J, Wang C, Zhang S, Pavlovicz RE, Jones A, Schmidt KH, Tang J, Dong H, Shan B, Fang B, Radhakrishnan R, Glazer PM, Matthias P, Koomen J, Seto E, Bepler G, Nicosia SV, Chen J, Li C, Gu L, Li GM, Bai W, Wang $\mathrm{H}$ and Zhang $\mathrm{X}$ : Hdac6 deacetylates and ubiquitinates msh2 to maintain proper levels of mutsalpha. Mol Cell 55(1): 31-46, 2014. PMID: 24882211. DOI: 10.1016/j.molcel.2014.04.028

25 Choi E, Lee C, Park JE, Seo JJ, Cho M, Kang JS, Kim HM, Park SK, Lee K and Han G: Structure and property based design, synthesis and biological evaluation of gamma-lactam based hdac inhibitors. Bioorg Med Chem Lett 21(4): 1218-1221, 2011. PMID: 21256006. DOI: 10.1016/j.bmcl.2010.12.079

26 Lee DH, Won HR, Ryu HW, Han JM and Kwon SH: The hdac6 inhibitor acy 1215 enhances the anticancer activity of oxaliplatin in colorectal cancer cells. Int J Oncol 53(2): 844-854, 2018. PMID: 29749542. DOI: 10.3892/ijo.2018.4405

27 Kwon S, Zhang Y and Matthias P: The deacetylase hdac6 is a novel critical component of stress granules involved in the stress response. Genes Dev 21(24): 3381-3394, 2007. PMID: 18079183. DOI: $10.1101 /$ gad.461107

28 Chou TC: Drug combination studies and their synergy quantification using the chou-talalay method. Cancer Res 70(2): 440-446, 2010. PMID: 20068163. DOI: 10.1158/0008-5472.CAN09-1947

29 Cahill DP, Levine KK, Betensky RA, Codd PJ, Romany CA, Reavie LB, Batchelor TT, Futreal PA, Stratton MR, Curry WT, Iafrate AJ and Louis DN: Loss of the mismatch repair protein msh6 in human glioblastomas is associated with tumor progression during temozolomide treatment. Clin Cancer Res 13(7): 2038-2045, 2007. PMID: 17404084. DOI: 10.1158/10780432.CCR-06-2149

30 Verhaak RG, Hoadley KA, Purdom E, Wang V, Qi Y, Wilkerson MD, Miller CR, Ding L, Golub T, Mesirov JP, Alexe G, Lawrence M, O'Kelly M, Tamayo P, Weir BA, Gabriel S, Winckler W, Gupta S, Jakkula L, Feiler HS, Hodgson JG, James CD, Sarkaria JN, Brennan C, Kahn A, Spellman PT, Wilson RK, Speed TP, Gray JW, Meyerson M, Getz G, Perou CM, Hayes $\mathrm{DN}$ and Cancer Genome Atlas Research N: Integrated genomic analysis identifies clinically relevant subtypes of glioblastoma characterized by abnormalities in pdgfra, idh1, egfr, and nf1. Cancer Cell 17(1): 98-110, 2010. PMID: 20129251. DOI: 10.1016/j.ccr.2009.12.020

31 Munoz JL, Rodriguez-Cruz V, Greco SJ, Ramkissoon SH, Ligon KL and Rameshwar P: Temozolomide resistance in glioblastoma cells occurs partly through epidermal growth factor receptormediated induction of connexin 43. Cell Death Dis 5: e1145, 2014. PMID: 24675463. DOI: 10.1038/cddis.2014.111

32 Ryu HW, Shin DH, Lee DH, Choi J, Han G, Lee KY and Kwon SH: Hdac6 deacetylates p53 at lysines $381 / 382$ and differentially 
coordinates p53-induced apoptosis. Cancer Lett 391: 162-171, 2017. PMID: 28153791. DOI: 10.1016/j.canlet.2017.01.033

33 Svechnikova I, Almqvist PM and Ekstrom TJ: Hdac inhibitors effectively induce cell type-specific differentiation in human glioblastoma cell lines of different origin. Int J Oncol 32(4): 821-827, 2008. PMID: 18360709.

34 Kovacs JJ, Murphy PJ, Gaillard S, Zhao X, Wu JT, Nicchitta CV, Yoshida M, Toft DO, Pratt WB and Yao TP: Hdac6 regulates hsp90 acetylation and chaperone-dependent activation of glucocorticoid receptor. Mol Cell 18(5): 601-607, 2005. PMID: 15916966. DOI: 10.1016/j.molcel.2005.04.021

35 Murphy PJ, Morishima Y, Kovacs JJ, Yao TP and Pratt WB: Regulation of the dynamics of hsp90 action on the glucocorticoid receptor by acetylation/deacetylation of the chaperone. J Biol Chem 280(40): 33792-33799, 2005. PMID: 16087666. DOI: 10.1074/jbc.M506997200

36 Lee SY: Temozolomide resistance in glioblastoma multiforme. Genes Dis 3(3): 198-210, 2016. PMID: 30258889. DOI: 10.1016/j.gendis.2016.04.007

37 Kondo S, Barnett GH, Hara H, Morimura T and Takeuchi J: $\mathrm{Mdm} 2$ protein confers the resistance of a human glioblastoma cell line to cisplatin-induced apoptosis. Oncogene 10(10): 20012006, 1995. PMID: 7761100.

38 Yip S, Miao J, Cahill DP, Iafrate AJ, Aldape K, Nutt CL and Louis DN: MSH6 mutations arise in GBM during temozolomide therapy and mediated temozolomide resistance. Clin Cancer Res 15(14): 4622-4629, 2009. PMID: 19584161. DOI: 10.1158/ 1078-0432.CCR-08-3012
39 Hunter C, Smith R, Cahill DP, Stephens P, Stevens C, Teague J, Greenman C, Edkins S, Bignell G, Davies H, O'Meara S, Parker A, Avis T, Barthorpe S, Brackenbury L, Buck G, Butler A, Clements J, Cole J, Dicks E, Forbes S, Gorton M, Gray K, Halliday K, Harrison R, Hills K, Hinton J, Jenkinson A, Jones D, Kosmidou V, Laman R, Lugg R, Menzies A, Perry J, Petty R, Raine K, Richardson D, Shepherd R, Small A, Solomon H, Tofts C, Varian J, West S, Widaa S, Yates A, Easton DF, Riggins G, Roy JE, Levine KK, Mueller W, Batchelor TT, Louis DN, Stratton MR, Futreal PA and Wooster R: A hypermutation phenotype and somatic msh6 mutations in recurrent human malignant gliomas after alkylator chemotherapy. Cancer Res 66(8): 3987-3991, 2006. PMID: 16618716. DOI: 10.1158/00085472.CAN-06-0127
Received November 12, 2019

Revised November 19, 2019

Accepted November 22, 2019 